\title{
Millisecond Pulsars in the Galactic Bulge?
}

\author{
An Extended Discussion on the Wavelet Analysis of the Fermi-LAT data \\ Richard Bartels and Christoph Weniger \\ GRAPPA Institute, University of Amsterdam, Science Park 904, 1090 GL Amsterdam, \\ Netherlands \\ email: r.t.bartels@uva.nl and c.weniger@uva.nl
}

\begin{abstract}
A clear excess in the Fermi-LAT data is present at energies around a few GeV. The spectrum of this so-called ' $\mathrm{GeV}$ excess' is remarkably similar to the expected annihilation signal of WIMP dark matter. However, a large bulge population of millisecond pulsars living below the Fermi-LAT detection threshold could also explain the excess spectrum. In a recent work we optimized the search for sub-threshold sources, by applying a wavelet transform to the FermiLAT gamma-ray data. In the Inner-Galaxy the wavelet signal is significantly enhanced, providing supportive evidence for the point source interpretation of the excess. In these proceedings we will extent our previous work with a spectral analysis and elaborate on the potential contamination from substructures in the gas.
\end{abstract}

Keywords. gamma-rays, pulsars: general, Galaxy: bulge, Galaxy: center

\section{Introduction}

For the past seven years the dark-matter (DM) community has been intrigued by a striking feature in the $\gamma$-ray spectrum of the Inner Galaxy. This so-called 'GeV-excess' peaks at energies around $2 \mathrm{GeV}$, appears spherically symmetric and is consisted with a contracted-NFW profile (Goodenough and Hooper (2009)). As such, it bares all the characteristics expected of a WIMP annihilation signal. Recently, the attention of the community has shifted from mostly characterising the excess (e.g. Daylan et al. (2016), Calore et al. (2015), Ajello et al. (2016)), to finding its origin. Apart from a DM explanation, other plausible scenarios include, amongst others, enhanced cosmic-ray injection in the Galactic Center (GC) (Carlson et al. (2016), Gaggero et al. (2015)) or the presence of a substantial population of sub-threshold point sources, most notably millisecond pulsars (MSPs) since their spectra resemble closely that of the excess. In the former scenarios the excess emission is inherently diffuse, whereas in the last it is point-like. Distinguishing between these two cases would provide a big step forward in understanding the origin of the excess. Two attempts to look for hints of unresolved point sources have recently been made, Lee et al. (2016) used non-Poissonian template fitting and Bartels et al. (2016) (henceforth BKW16) analysed local-maxima in the wavelet-transformed skymap. Both works find enhanced clustering of photons, providing corroborative evidence for the presence of a yet unresolved central source population (CSP) with plausibly sufficient numbers to explain $100 \%$ of the excess $\gamma$-rays.

Assuming a MSP $\gamma$-ray luminosity function $d N / d L_{\gamma} \propto L_{\gamma}^{-1.5}$ and a spatial distribution $\rho_{\mathrm{MSP}}(r) \propto r^{-2.5}$ extending out to $3 \mathrm{kpc}$, the results of BKW16 indicated that $100 \%$ of 

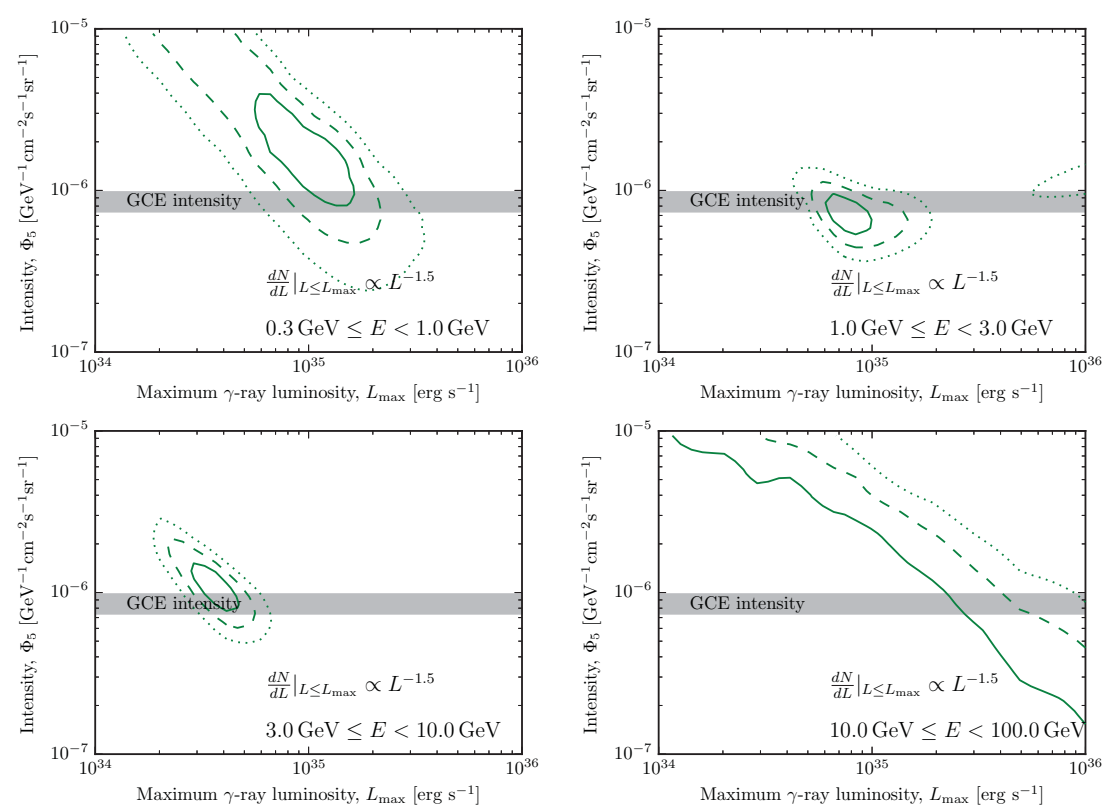

Figure 1. Constraints on the maximum $\gamma$-ray luminosity of the CSP, $L_{\max }$, and the population averaged intensity $\left(\Phi_{5}\right)$ at $b= \pm 5^{\circ}, \ell=0^{\circ}$ and $2 \mathrm{GeV}$ for analyses in different energy bins (see inset). Contours are for $68.7 \%, 95.4 \%$, and $99.7 \%$ C.L.

the GeV-excess can be explained by a MSP population with a maximum luminosity of $L_{\gamma, \max }=7 \times 10^{34} \mathrm{erg} \mathrm{s}^{-1}$ and $\sim 3000(35000)$ MSPs above $L_{\gamma} \geqslant 10^{33}\left(10^{31}\right) \mathrm{erg} \mathrm{s}^{-1}$. $\dagger$

For more details on the original analysis we refer the reader to BKW16. Below we extent the discussion of the wavelet results by discussing a spectral analysis in Sect. 2 and by elaborating on the potential impact of small-scale gas structures on our results in Sect. 3 .

\section{Spectral results}

The original wavelet analysis by Bartels et al. (2016) was performed in a single energy bin, 1-4 GeV. We redo the analysis using almost seven years of Fermi-LAT P8R2 source class data in 4 bins, respectively $0.3-1,1-3,3-10$ and 10-100 GeV. For the MSP spectrum used in the Monte-Carlo simulations (MCs) we assume $d N / d E \propto e^{-E / 3.78 \mathrm{GeV}} E^{-1.57}$, as was found to give a good fit to a stacked sample of MSP spectra by Cholis et al. (2014). The results of this analysis are shown in Fig. 1.

Only in the 1-3 and 3-10 GeV bins do we find a strong preference for an additional point source like component on top of the diffuse emission, at respectively $9.2 \sigma$ and $10.4 \sigma$. In the lowest energy band $(0.3-1 \mathrm{GeV})$ the results are weaker, but additional clustering of photons is still formally preferred compared to diffuse only emission at the level of $\sim 5 \sigma$. However, the analysis in this bin might be compromised by the size of the PSF. In the highest band $(10-100 \mathrm{GeV})$ the lack of photons limits the analysis and we only

$\dagger$ In light of the recent discovery of a magnetar in the GC (e.g. Eatough et al. (2013)) and because of general interest of the community in high-precision clocks in this region, we extrapolate the best-fit population for the assumed luminosity and spatial distributions down to the GC. This leads to $\sim 60(600)$ MSPs above $L_{\gamma} \geqslant 10^{33}\left(10^{31}\right) \mathrm{erg} \mathrm{s}^{-1}$ within the inner 1 pc. The radio counterparts of these hypothetical $\gamma$-ray emitting MSPs at the GC could potentially be useful in tests of general relativity. 

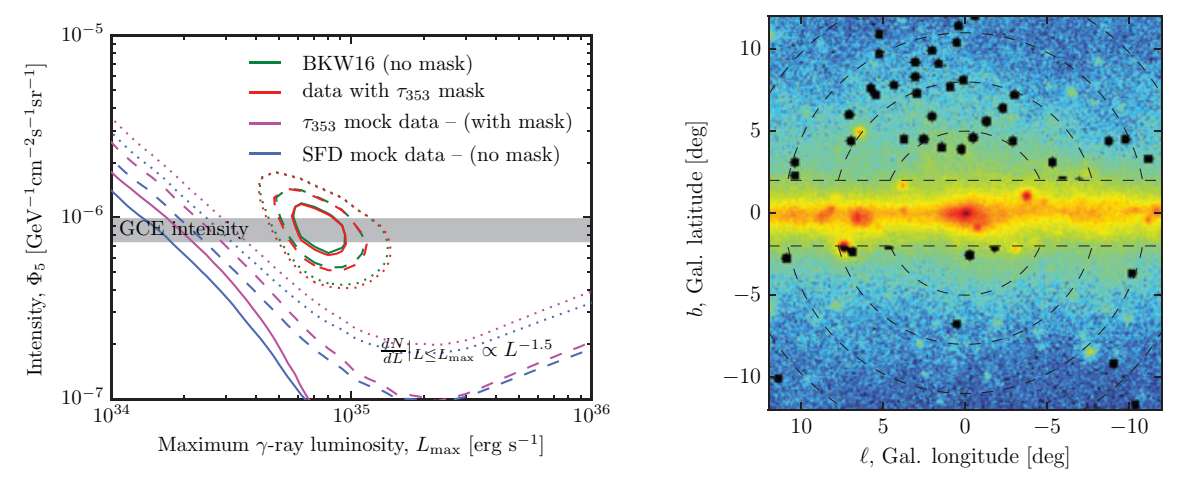

Figure 2. Left: The results from BKW16 and contours for the same data, but with the Planck $\tau_{353}$ inspired mask for the gas applied. We also show upper limits that are obtained from the the $\tau_{353}$ /SFD mock data sets. To the $\tau_{353}$ mock data we apply the same mask as is applied to data, this removes all structure yielding a substantial wavelet signal from the mock map. Right: count map of the inner galaxy (log scaled), with the mask based on the Planck optical depth map superimposed (black).

find an upper limitł. Note that for these results we masked all 3FGL sources, apart from 13 that were also left unmasked in the original BKW16 work. The results do not critically hinge on the (un)masking of these 13 sources. Finally, we confirmed that there is a spatial correlation for a large fraction of the most significant wavelet peaks (with all 3FGL sources masked in this case) in the 1-3 GeV or 3-10 GeV energy bins. A similar correlation of peaks is not found between any other two sets of energy ranges.

\section{Small scale gas emission}

The Fermi-LAT diffuse models are smoothed on a $1^{\circ}$ scale, and therefore by construction do not capture the small-scale features of the gas. This raises the question whether small-scale structures in the gas could potentially give rise to the wavelet signal. As a possible caveat, it was pointed out in the supplemental material of BKW16 that molecular clouds of $\mathcal{O}\left(\right.$ few $\left.\times 10^{5} \mathrm{M}_{\odot}\right)$ can potentially give rise to a $\gamma$-ray signal required to explain the brightest wavelet peaks. However, this scenario we consider to be unlikely since such clouds would have to be transported up to high latitudes $(>1 \mathrm{kpc}$ from the disk) and it is not obvious why these clouds would only be present in the Inner-Galaxy and not along the disk. We remind the reader that our wavelet signal is purely a feature of the Inner-Galaxy (see supplemental material of BKW16).

Nevertheless, we perform an additional check which illustrates that it is unlikely that our wavelet signal is fully caused by gas emission. We take two different tracers for the gas, the Planck dust optical depth map at $353 \mathrm{MHz}\left(\tau_{353}\right)$ (Abergel et al. $(2014) \S$ and the dust map by Schlegel et al. (1998) (henceforth, SFD). The aim is to use these maps as an alternative template for diffuse emission. For this purpose, they are smoothed with the Fermi-LAT point-spread function in the $1-4 \mathrm{GeV}$ band (since the aforementioned maps have resolutions $\lesssim 0.1^{\circ}$ ) and scaled to the intensity of the diffuse-emission model from Fermi (gll_iem_v06.fits) at $|b| \geqslant 2^{\circ}$.

In particular, the $\tau_{353}$ inspired diffuse model contains more structure than the Fermi

$\ddagger$ There is a total of one wavelet peak with $\mathcal{S} \geqslant 3$ in this energy range. This peaks is masked to make the plot clearer. The impact of the masking does not affect the results.

$\S$ The proportionality between the optical depth and the gas column density depends on the opacity $\left(\sigma_{353}\right), \tau_{353}=\sigma_{353} N_{\mathrm{H}}$. 
diffuse models. Rerunning our analysis, but replacing the observational data by mock data from MC realizations of the $\tau_{353}$ diffuse template plus noise, we find an enhanced wavelet signal compared to the predictions from our MCs of the Fermi diffuse model only. $\uparrow$ To remove the impact of these additional peaks we construct a mask of $0.3^{\circ}$ radius at the position of all $\mathcal{S} \geqslant 1$ wavelet peaks present in the wavelet-transformed map of the $\tau_{353}$ template without Poisson noise. The mask is shown in the right plot of Fig. 2. Applying this mask to our mock-data run now yields a result consistent with no additional photon clustering, meaning that we effectively removed the gas-induced wavelet peaks in this template. On the other hand, if we apply this mask to the Fermi-LAT data we obtain a best-fit contour that only marginally differs from our original result, implying that also after using the dust-inspired masks there is a clear indication of an additional source of wavelet peaks. The results are shown in the left panel of Fig. 2.

\section{Conclusion}

The clustering of photons as detected by the wavelet analysis provides a strong indication for the presence of a CSP below the Fermi-LAT detection threshold. The enhanced wavelet signal is most strongly detected in the GeV excess energy window. Wavelet analyses of existing dust maps, used here as a tracer of the gas, seem to imply that there is not enough small-scale structure in the gas to explain the enhanced signal in the Galactic centre. This favours the interpretation of a CSP as the source of the photon clusters. Finally, $\gamma$-rays are unlikely to provide conclusive evidence in favour of either the pointsource interpretation or any other origin of the excess. The MSP scenario will, however, soon be tested by SKA and its pathfinder, Meerkat (Calore et al. (2016)).

\section{Acknowledgements}

RB wishes to thank the organizers and participants of the IAU symposium 322, Palm Cove, Australia, 18-22 July, for an inspiring conference. This research is supported by NWO through a PhD fellowship (RB) and a Vidi grant (CW).

\section{References}

Abergel, A. et al. 2014, A\&A, 571, A11

Ajello, M. et al. 2016, ApJ, 819, 44

Bartels, R., Krishnamurthy, S., \& Weniger, C. 2016, Phys. Rev. Lett., 116, 051102

Calore, F., Cholis, I., \& Weniger, C. 2015, JCAP, 1503, 038

Calore, F., Di Mauro, M., Donato, F., \& Hessels, J. W. T. and Weniger, C. 2016, ApJ., 827, 143

Carlson, E., Linden, T., \& Profumo, S. 2016, Phys. Rev. Lett., 117, 111101

Cholis, I., Hooper, D., \& Linden, T. 2014, arXiv:1407.5583

Daylan, T., Finkbeiner, D. P., Hooper, D., Linden, T., Portillo, S. K. N., Rodd, N. L., \& Slatyer, T. R. 2016, Phys. Dark Univ., 12, 1-23

Eatough, R. P. et al. 2013, Nature, 501, 391-394

Gaggero, D., Taoso, M., Urbano, A., Valli, M., \& Ullio, P. 2015, JCAP, 1512, 056

Goodenough, L. \& Hooper, D. 2009, arXiv:0910.2998

Lee, S. K., Lisanti, M., Safdi, B. R., Slatyer, T. R., \& Xue, W. 2016, Phys. Rev. Lett., 116, 051103

Schlegel, D. J., Finkbeiner, D. P., \& Davis, M. 1998, ApJ, 500, 525

ब Performing the same check with the SFD template yields a result consistent with no additional source of wavelet peaks. 